\title{
It's About Time: Towards a Longitudinal Cognitive Neuroscience of Intelligence
}

\author{
Rogier A. Kievit \\ Ivan L. Simpson-Kent
}

\section{Introduction}

The search for the biological properties that underlie intelligent behaviour has held the scientific imagination at least since the pre-Socratic philosophers. Early hypotheses posited a crucial role for the heart (Aristotle; Gross, 1995), the ventricles (Galen; Rocca, 2009), and the 'Heat, Moisture, and Driness' of the brain (Huarte, 1594). The advent of neuroimaging technology such as EEG, MEG and MRI has provided more suitable tools to scientifically study the relationship between mind and brain. To date, many hundreds of studies have examined the association between brain structure and function on the one hand and individual differences in general cognitive abilities such as intelligence on the other. Both qualitative and quantitative reviews have summarized the cross-sectional associations between intelligence and brain volume (Pietschnig, Penke, Wicherts, Zeiler, \& Voracek, 2015), as well as more network- and imaging-specific hypotheses which suggest a key role for the frontoparietal system in supporting individual differences in intelligence (Basten, Hilger, \& Fiebach, 2015; Deary, Penke, \& Johnson, 2010; Jung \& Haier, 2007). These findings are bolstered by converging evidence from lesion studies (Barbey, Colom, Paul, \& Grafman, 2014), cognitive abilities in disorders associated with physiological abnormalities (Kail, 1998) and the neural signatures associated with the rapid acquisition of new skills (Bengtsson et al., 2005).

These innovations in neuroimaging coincided with the emergence of more dynamic, longitudinal models of the development of intelligence. Where seminal works on intelligence such as Spearman (Spearman, 1904) and Jensens' The g Factor: The Science of Mental Ability (Jensen, 1998) barely discuss developmental change, new theories have begun to address the role of development to understand intelligence conceptually and empirically. For instance, theories such as that of Dickens \& Flynn (Dickens \& Flynn, 2001) suggest direct, reciprocal interactions between intelligence, genetic predisposition and the environment over the lifespan. This model, where genetic predispositions lead to people self-stratifying to environments in line with their abilities, leads to amplification of initial differences, thus reconciling previously puzzling facts about heritability and environmental influences. Later, inspired by ecological models of predator-prey relationships, van der Maas (2006) proposed the mutualism model, which suggests that general cognitive ability emerges, at least in part, due to positive reciprocal influences between lower cognitive faculties. In other words, greater ability in one domain, such as vocabulary, may facilitate faster growth in others (memory, reasoning) through a range of mechanisms. Recent empirical studies (Ferrer \& McArdle, 2004; Kievit, Hofman, \& Nation, 2019; Kievit et al., 2017) in longitudinal samples as well as meta-analytic and narrative reviews (Peng \& Kievit, 2019; Peng, Wang, Wang, \& Lin, 2019) find support for the mutualism model, suggesting a key role for developmental dynamics in understanding cognitive ability. Converging evidence for the plausibility of such 
dynamic models comes from atypical populations. For instance, Ferrer, Shaywitz, Holahan, Marchione, \& Shaywitz (2010) demonstrated that a subpopulation with dyslexia was characterized not (just) by differences in absolute performance in reading ability, but by an absence of positive, reciprocal effects between $I Q$ and reading compared to typical controls.

However, there is very little work on the intersection between these two innovative strands of intelligence research: How changes in brain structure and function go hand in hand with changes in cognitive abilities associated with intelligence. This is unfortunate, as to truly understand the nature of the relationship between emerging brains and minds, there is no substitute for longitudinal data (Raz \& Lindenberger, 2011), where the same individuals undergo repeated sessions of neuroimaging as well as repeated assessments of higher cognitive abilities closely associated with intelligence.

\section{Towards a dynamic cognitive neuroscience of intelligence}

Our goal in this chapter is to examine longitudinal studies that study change in both cognitive function and brain structure in childhood, adolescence and early adulthood, when change in both domains is rapid. A similarly exciting question is neurocognitive ageing at the other end of the lifespan - However, as that has recently been comprehensively reviewed (Oschwald et al., 2019), this chapter will focus on the period from early childhood to early adulthood. We will focus (with some partial exceptions) on studies that measure both cognitive ability and measures of brain structure on at least two time points. Such studies are sufficiently rare that we can survey them here comprehensively. Although we focus on intelligence, we do not limit ourselves to studies that use IQ scores, but rather studies that measure continuous measures of cognitive ability that are canonically considered closely related to intelligence (e.g. as defined by high standardised factor loadings in a hierarchical factor model). These include measures such as (working) memory, fluid reasoning, vocabulary and processing speed, all of which generally show steep developmental increases, and as such are likely most sensitive to contemporaneous brain changes.

To understand the unfolding dynamics between cognitive and neural change requires longitudinal data as well as longitudinal methodology. As outlined in Kievit et al., 2018 (see Figure 1), we can conceptualise the relationship between brain change and cognitive change in terms of three key parameters: Brain structure driving cognitive change, cognitive ability leading to brain reorganization, and correlated change.

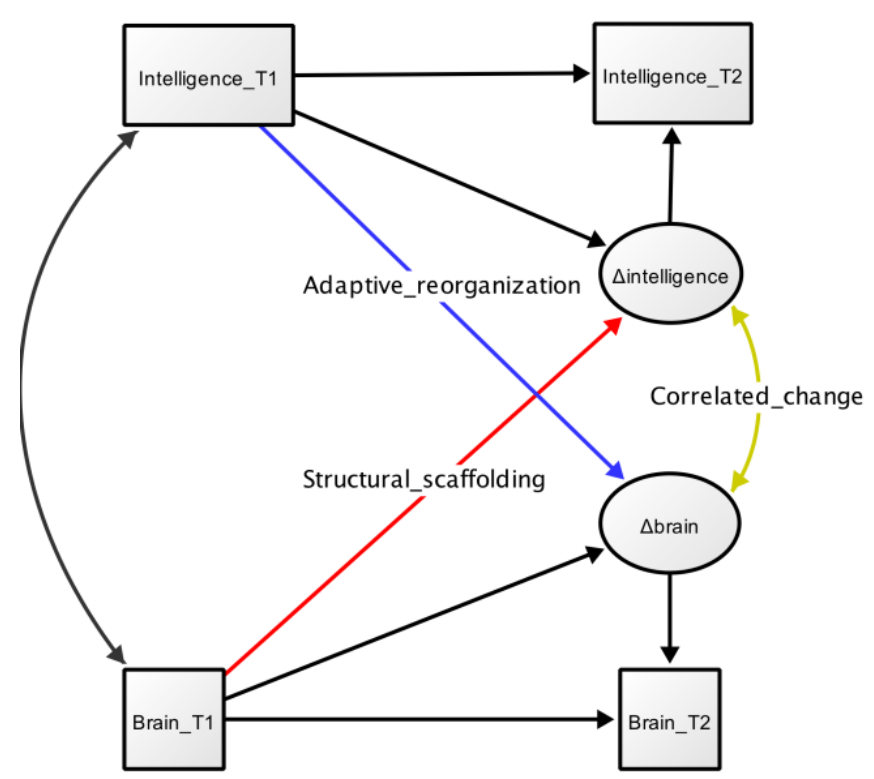

Figure 1. Simplified bivariate latent change score model illustrating the co-development of intelligence scores (top) and brain measures (bottom) across two waves. For more details, see Kievit et al., (2018).

First, current brain structure may be found to govern the rate of change in cognitive 
performance. This is what we would refer to as 'structural scaffolding'. According to this hypothesis, the current state of the brain (indexed either structural or functional measures) provides, in some sense, the preconditions that facilitate cognitive growth. Specifically, we would expect that individuals with more optimal neural characteristics (e.g. high volume, greater white matter integrity etc.) would show larger rates of cognitive gain. This effect can be quantified by means of a coupling parameter in a latent change score (LCS) model, a regression that quantifies the association between the current state of brain structure on the rate of change (delta) of the cognitive domain of interest (red, upward arrow in Figure 1). Alternatively, current cognitive performance may be associated with the rate of change of structural (or functional) brain metrics. This could be conceptualized as cognitive plasticity, or reorganization. For instance, achieving a certain greater level of cognitive ability may lead to more rapid reorganization of cortical structure to engrain, or solidify, these newly acquired abilities. Recent mechanistic proposals (Wenger, Brozzoli, Lindenberger, \& Lövdén, 2017) have shown how a mechanistic cascade of glial changes, dendritic branching and axonal sprouting following rapid skill acquisition lead to volume expansion, followed by a period of renormalization. Such effects can be captured by a coupling parameter in an LCS model (blue, downward arrow in Figure 1). Both these parameters can be further expanded such that the recent rate of change in one domain governs future changes in another domain (Estrada, Ferrer, Román, Karama, \& Colom, 2019; Grimm, An, McArdle, Zonderman, \& Resnick, 2012). Finally, changes in brain structure and cognitive function may be correlated (yellow doubleheaded arrow in Figure 1). Although not all analytical approaches allow for the investigation of all these parameters, and the papers we discuss below show considerable methodological heterogeneity, we find the above a fruitful way to frame how associations over time unfold. We will discuss the findings as presented, and present suggestions at the end of this chapter for future work. The empirical papers below are described in more detail (sample sizes, age range, measures of interest) in Table 1, and an at-a-glance overview of the age ranges and number of occasions is illustrated in Figure 2.

\section{Grey matter}

One of the earliest papers, Sowell et al. (2004), focused on mapping cortical changes across two years in 45 children ages 5-11 years. Greater cortical thinning was associated with more rapid gains in vocabulary, especially in the left hemisphere. A considerably larger study by Shaw et al. (2006) grouped 307 children into three 'strata' of intelligence (high, medium and low) and observed a complex pattern. High ability children showed especially pronounced changes, with early steep increases in volume followed by steeper rates of cortical thinning afterwards. Notably, this differential process itself induced different brain-intelligence associations, with negative associations between cortical thickness and intelligence at early ages but positive associations later on in development. Future work in (very) large samples, such as the Adolescent Brain Cognitive Development (ABCD) study (Volkow et al., 2018), is uniquely positioned to examine the replicability and consistency of these early findings.

A more recent follow-up study attempted to tease apart cognitive specificity for such associations. Ramsden et al. (2011) examined longitudinal changes in verbal and non-verbal 
intelligence in a relatively small sample $(\mathrm{N}=33)$ of healthy adolescent participants and their associations with grey matter. Correlating change scores across two measurements, three years apart, the authors showed that changes in verbal intelligence (VIQ) co-occurred alongside changes in grey matter density in a region of the left motor cortex previously linked to articulation of speech (Ramsden et al., 2011, pp. 114). In contrast, changes in non-verbal intelligence were positively correlated with grey matter density in the anterior cerebellum, which has previously been implicated in hand motor movements. Although preliminary, this work suggests potential specificity in neurodevelopmental patterns. Burgaleta, Johnson, Waber, Colom, and Karama (2014) examined changes in intelligence test scores across two waves and observed correlated change between cortical thickness (especially in frontoparietal areas) and changes in intelligence. The pattern of results showed that those with greatest gains in FSIQ showed less rapid cortical thinning than those with smaller gains, or decreases, in FSIQ. Similar results were not observed for cortical surface area, suggesting greater sensitivity of thickness to cognitive changes.

Schnack et al. (2015) expanded beyond only children to a wider, lifespan sample (960), although weighted towards children and adolescents. As observed in Shaw et al. (2006), they observed a developmentally heterogeneous set of associations, with thinner cortices being associated with better cognitive performance at age 10, and high IQ children showing more rapid thinning. However, in adulthood this pattern reversed, such that greater cortical thickness in middle age is associated with higher intelligence, possibly due to slower lifespan thinning, further emphasizing the importance of a truly longitudinal, developmental perspective.

The majority of work focuses on thickness, volume and area of cortical regions. In contrast, Tamnes, Bos, van de Kamp, Peters, and Crone (2018) studied longitudinal changes in the hippocampus and its subregions in 237 individuals scanned up to three times. They observed cross-sectional correlations between intelligence and hippocampal subregions, but the only significant longitudinal associations were a positive association between the rates of increase in the molecular layer of the hippocampus and cognitive performance. Interestingly, such correlated changes may be specific to certain subtests of intelligence, even those considered quite central to cognitive ability such as working memory: Tamnes et al., (2013) showed that greater volume reductions in the frontal and rostral middle frontal gyri were associated with greater gains in working memory, even after adjusting for $\mathrm{IQ}$.

Although most studies focus on properties of brain structure directly (e.g. thickness, volume), an emerging subfield focuses on the covariance between regions instead. Khundrakpam et al. (2017) used structural covariance between regions to characterize the covariance network between regions. In 306 subjects scanned up to three times, they observed greater cortical thickness, higher global efficiency but lower local efficiency in individuals with higher IQ, especially in frontal and temporal gyri. Although based on longitudinal data, the rate of change itself was not directly used in this study.

Where most studies use summary metrics of intelligence or cognitive performance, increasingly authors implement full (longitudinal) latent variable models (Román et al., 2018). The benefits of doing so are many, including increased power, establishment of measurement 
invariance, which allows for unbiased interpretation of change over time (Widaman, Ferrer, \& Conger, 2010), and more flexibility regarding missing data.

Ritchie et al. examined a large ( $N=2316)$ number of adolescents (14-19) tested and scanned in two waves, approximately five years apart from the IMAGEN sample. Ritchie et al. examined the relationship between (changes in) a broad general of ability (by extracting the first component of a battery of CANTAB tasks) and (changes in) a global (cortical) summary of grey matter, indexed by volume, thickness and surface area. Using a Latent Change Score modelling strategy, they observed constellation of interesting patterns. Cross-sectionally, higher cognitive ability was correlated with higher cortical volume and larger surface area, with weaker results for cortical thickness. Those with higher baseline ability tended to show more rapid cortical thinning and volume loss, although this was a relatively small effect. In contrast with some other findings, baseline brain structure was not associated with rates of cognitive change over time.

Román et al. studied longitudinal changes in a measurement invariant g factor alongside changes in cortical thickness and surface area in a sample of 132 children and adolescents (6 to 21 years) from the NIH Paediatric MRI Data Repository (Evans, 2006). A general intelligence factor was estimated at 3 time points with an average interval of 2 years. Changes in g scores correlated with changes in cortical thickness as well surface area $(r=.3 / .37)$, in all regions (for cortical thickness) but mainly fronto-temporal (for surface area). Moreover, the trajectories of cortical thinning depended on cognitive ability: Significant cortical thinning was apparent at age 10-14 for individuals with lower g scores, whereas for those with higher g scores, cortical thinning only became apparent around age 17. A followup study on the same sample by a similar team of authors extended the analysis to what is likely the most advanced psychometric analysis in this field to date (Estrada et al., 2019). Using Latent Change Score models in 3 waves, the authors were able to tease apart lead-lag relations between intelligence and brain structure. Notably, and unlike any other paper to date, they used the estimates of current rates of change (at wave 2) to predict future rates of change in the other domains (see also Grimm, An, McArdle, Zonderman, \& Resnick, 2012 for a technical overview). They observed a complex but fascinating pattern of results: Although changes in cognitive ability or cortical structure were not predicted by the level in the other domain one time point before, the recent rate of change did predict future changes. In other words, individuals who showed less thinning and less surface loss showed greater gains in general intelligence in the subsequent period. In contrast, individuals who increased more in g during the previous period showed greater subsequent thinning - Possibly due to greater reorganization following the cognitive skill gains. For all analyses surface area showed less pronounced effects, suggesting, as in other studies, that cortical thickness is a more sensitive measure than surface area. These findings offer intriguing insights into the true intricacies of the unfolding development of cognition and brain structure, and suggest that even higher temporal resolution, as well as greater numbers of measurement occasions, are needed to truly capture these processes.

The majority of studies focused on children, usually from age 7-8 onwards, likely for practical and logistical reasons. However, exceptions exist. Jaekel, Sorg, Baeuml, Bartmann, \& 
Wolke, 2019) studied the association between head growth and intelligence in 411 very preterm, preterm and term born infants. Doing so, they observed that greater perinatal head size, as well as faster head growth (especially in the first 20 months) were associated with better cognitive performance, together explaining up to $70 \%$ of the variance in adult $I Q$. Other infant studies focusing on white matter will be discussed below.

\section{White matter}

Much of the initial work on longitudinal studies of intelligence focused on grey matter structure, especially volume and thickness. In recent years, technology, especially quantitative models, to capture and quantify white matter microstructure have made considerable strides. Most of these innovations have focused on diffusion weighted imaging (DWI), which allows researchers to capture metrics such as fractional anisotropy (FA), mean diffusivity (MD) and neurite dispersion. Although the mapping from such measures to the underlying physiology such as axonal width and myelination remains far from perfect (Jones, Knösche, \& Turner, 2013; Wandell, 2016), DWI measures have provided a range of new insights into the development of intelligence.

Ferrer (2018) examined developmental changes in fluid reasoning in an $N=201$, three wave sample of children, adolescents, and young adults ages 5-21 (Earlier work by the same group relies on lower numbers from the same sample; Ferrer et al., 2013). Fluid reasoning was assessed using Matrix Reasoning, Block Design, Concept Formation and Analysis Synthesis. Notably, Ferrer incorporated both a latent variable of fluid reasoning, as well as establishing measurement invariance across waves. Ferrer observed that greater global white matter volume (in $\mathrm{mm}$ ) and greater white matter microstructure (indexed as fractional anisotropy) were associated with more rapid improvements in fluid reasoning. However, white matter was only incorporated at baseline, precluding the examination of cognitive performance driving white matter change.

In a small cohort of children scanned on two occasions ( $N=37$, age range: 6 to 8 years, two year interval between scans), Borchers et al., 2019 examined the influence of white matter microstructure (mean tract-FA) at age 6 on subsequent reading ability (Oral Reading Index) at age 8. Reading ability was assessed at age 6 (considered by the authors as the onset of learning to read), with concurrent estimates of mean tract-FA of tracts known to be involved in reading-related abilities. They found that age 8 reading ability was predicted by mean tract-FA of the left inferior cerebellar peduncle as well as left and right superior longitudinal fasciculus even after controlling for age 6 pre-literacy skills and demographic indicators (family history \& sex).

Moving beyond summary metrics, Koenis et al. (2015) examined within-subject white matter networks using mean FA as well as streamline count, and computed graph theory metrics such as global and local efficiency (both network metrics, which quantify the ease with which two nodes in a network can reach each other through edges, or 'connections') to characterize the nature of the within-subject networks. They demonstrated that children who made the greatest gains in efficiency (not to be confused with 'neural efficiency' during task performance, see Neubauer \& Fink, 2009) of their structural network were those who made 
the greatest gains in intelligence test scores. In contrast, individuals who showed no change or a decrease in network efficiency showed a decrease in intelligence test scores. The strongest nodal associations were present for orbitofrontal cortex and the anterior cingulum. The associations for streamline, rather than FA, based networks were largely non-significant or marginally so - but sometimes inconsistent with FA based efficiency.

Two studies examined the role of white matter microstructure in infants. Young et al. (2017) examined the white matter trajectories (as quantified by FA, MD, AD and RD) in 75 very preterm neonates across up to 4 waves of diffusion weighted imaging. Doing so, they observed that slower decrease in mean diffusivity (defined by MD) was associated with lower full scale IQ scores later on in life. Moreover, Deoni et al. (2016) studied the myelination profiles of 257 healthy developing children. A subset ( $N=126)$ was scanned and tested at least twice, with further subsamples being scanned up to 5 times to derive advanced myelination metrics such as myelin water fraction. In all regions studied, children of above average cognitive ability showed distinct myelination trajectories: Higher intelligence test scores were associated with a longer initial lag and slower growth period, followed by a longer overall growth phase and faster secondary growth rates, yielding the most pronounced crosssectional differences at age 3 .

\section{The role of genetics}

As shown above, an emerging body of work suggests key roles for cortical and subcortical maturation in supporting changes in intelligence. However, these observations leave open the question of the aetiology of these processes. Are they driven largely by concurrent changes and differences in the environment? Or do underlying genetic differences underlie most or all of the observed processes? To address these questions, , Schmitt et al. (2019) used a twin design to examine the associations between (changes in) cortical thickness and baseline intelligence, as well as the extent to which they shared genetic similarity, in 813 typically developing children with up to as many as 8 scans. Using a twin analysis, the authors showed that the phenotypic covariance between IQ and cortical thickness and cortical thickness change was effectively entirely genetic. Koenis et al. (2018) showed (in the same sample as Koenis et al., 2015 above) age-dependent correlations between brain measures and IQ: Weak or absent correlations early in childhood ( $r=0$ at age 10) became pronounced by middle to late adolescence (age $18, r=.23$ ). Notably, the previous steep changes in efficiency between age 10 and 13 had levelled off by age 18, suggesting a slowing down of cortical development. Finally, a unique twin design allowed the authors to compute genetic correlations: The extent to which brain network changes and intelligence changes may share an underlying genetic origin. They observed a steady increase in this genetic correlation, suggesting that developmental trajectories continue to unfold, an interpretation in line (in spirit) with Dickens-Flynn type models of development, where genetic predispositions lead to an increasingly close alignment of genetic predispositions and the environment.

Brans et al. (2010) investigated changes in cortical thickness in 66 twins (total $N=132$ ) in late adolescence and adults (20-40 years) scanned on two occasions. They demonstrated, as did studies above, that individuals with higher IQ's (defined by full WAIS-III) showed greater 
thickening and less thinning than those with lower IQ's. Notably, the genetic correlations where significantly different between those affecting current thickness versus rate of thinning, suggesting distinct aetiological pathways between the state of the brain and its developmental pathway. The genetic analysis showed moderate to strong, regionally specific, genetic covariance between the rate of thickness change and the level of intelligence.

A recent study in the IMAGEN sample, ( $N=551$, Judd et al. (2020), age 14/19) examined the role of SES and a polygenic risk score of educational attainment (summarized across thousands of genetic loci) in the development of brain structure and working memory. The core analysis centred on the global effects of SES on the brain, but more specific effects of the polygenic risk score on individual differences in brain structure. For our purposes, the key finding was the bivariate latent change score model, which showed that individuals with higher baseline working memory ability showed a stronger decrease in global surface area during adolescence. In contrast, differences in baseline surface area at age 14 was not associated with differences in the rate of working memory improvement - although the authors note a potential performance ceiling effect limits strong conclusions. Polygenic risk scores did not differentially predict the rate of surface area change.

\section{Other imaging measures}

Although the majority of the work on the development of intelligence relies on structural and functional MRI, some exceptions exist. An extremely early study (Beckwith \& Parmelee, 1986) examined sleep-related EEG markers and intelligence in 53 infants. Preterm infants who displayed a particular EEG pattern ('trace alternant') during (transitional) sleep showed better intelligence scores at age 8. Hahn et al. (2019) studied longitudinal changes in sleep spindles based on polysomnography (EEG recording during sleep) in 34 children across a 7-year interval, to quantify slow and fast sleep spindle power. They observed interesting but preliminary findings such that individuals with higher cognitive ability showed greater increase in frontal slow spindle activity. Together these two studies suggest that neural activity during sleep is associated with the level and change of intelligence.

Although most studies focus on a single neuroimaging metric, more recent work has incorporated both functional and structural connectivity. Evans et al. (2015) examined 79 children (43 with scans) alongside changes in the Wechsler Abbreviated Scale of Intelligence, with a special focus on numerical operations. They showed that greater grey matter volume at baseline, especially in prefrontal regions, was associated with greater gains in numerical operations over time. In contrast, functional connectivity in, and between, the same regions identified in the grey matter volume analysis were not associated with greater gains in numerical abilities. A more recent study arrived at a similar conclusion: Wendelken et al. (2017) measured fluid intelligence as well as functional connectivity (FC) and structural connectivity (SC, defined as mean fractional anisotropy in tracts connecting key regions) and related this to changes in reasoning from childhood to early adulthood (age range: 6-22 years). Interestingly, this study incorporated pooled data from three datasets (some included in other papers reported here) with reasoning, FC, and/or SC data for at least two time points. 
The aggregate sample consisted of 523 participants. Cross-sectional analysis revealed differential age relations between FC, SC and reasoning. Specifically, SC was strongly (and positively) related to reasoning ability in children but not adolescents and adults. In adolescents and adults, FC was positively associated with reasoning, but this effect was not found in children. Longitudinal analyses revealed fronto-parietal (RLPFC-IPL) SC at one time point positively predicted RLPFC-IPL FC, but not vice versa. Moreover, in young participants (children), SC was positively associated with change in reasoning. Together, these findings suggest that brain structure, especially white matter, may be a stronger determinant of longitudinal cognitive change than functional connectivity. 


\begin{tabular}{|l|}
\hline Beckwith \& Parmelee, 1986 \\
\hline Sowell et al., 2004 \\
\hline Shaw et al., 2006 \\
\hline Brans et al., 2010 \\
\hline Ramsden et al., 2011 \\
\hline Tamnes et al., 2013 \\
\hline Burgaleta et al., 2014 \\
\hline Evans et al., 2015 \\
\hline Koenis et al., 2015 \\
\hline Schnack et al., 2015 \\
\hline Deoni et al., 2016 \\
\hline Wendelken et al., 2017 \\
\hline Khundrakpam et al., 2017 \\
\hline Young et al., 2017 \\
\hline Koenis et al., 2018 \\
\hline Román et al., 2018 \\
\hline Tamnes et al., 2018 \\
\hline Ferrer, 2018 \\
\hline Jaekel et al., 2019 \\
\hline Estrada et al., 2019 \\
\hline Schmitt et al., 2019 \\
\hline Hahn et al., 2019 \\
\hline
\end{tabular}

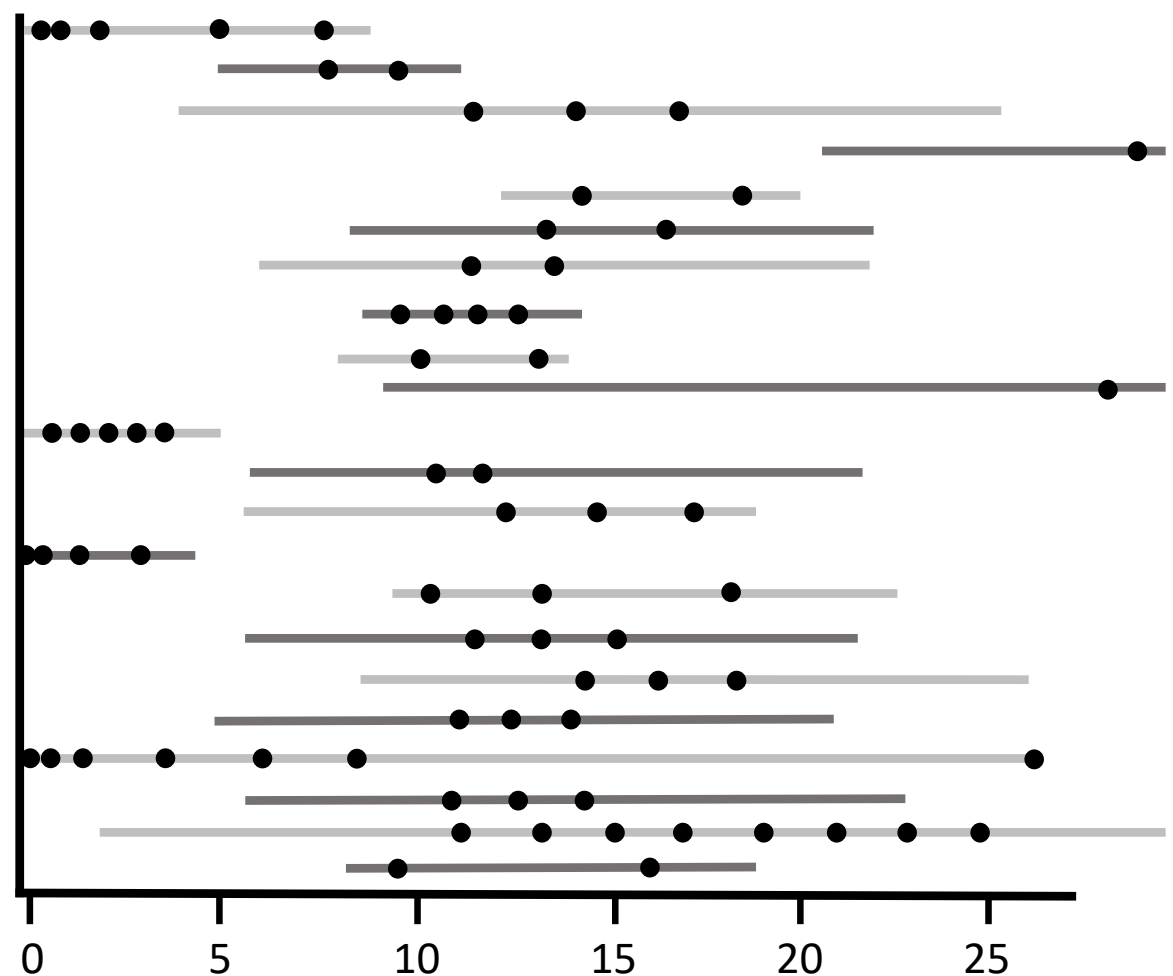

Figure 2. An overview of longitudinal studies of brain structure, function and intelligence. For each study, organized chronologically, we show the age range (lines), number of waves (number of dots) and mean age at each wave (location of dots). Lines that extend beyond the graph rightwards have more waves beyond early adulthood. More details per study are shown in the table below.

\begin{tabular}{|c|c|c|c|c|c|c|}
\hline Reference & $\begin{array}{c}\text { Publication } \\
\text { year }\end{array}$ & $\begin{array}{l}\text { Sample size (per } \\
\text { wave) }\end{array}$ & $\begin{array}{l}\text { between waves } \\
\text { (years) }\end{array}$ & Cognitive test(s) & Imaging metric & $\begin{array}{l}\text { Age range } \\
\text { (years) }\end{array}$ \\
\hline $\begin{array}{l}\text { Beckwith \& Parmelee, } \\
1986\end{array}$ & 1986 & $53 / 53 / 53 / 53 / 49$ & $\sim 1$ & $\begin{array}{l}\text { Gesell Developmental Scale (4, 9, } 24 \mathrm{mo} \text { ), } \\
\text { Stanford-Binet Intelligence Scale (age 5-8), } \\
\text { WISC (age 8) }\end{array}$ & Electroencephalogram (EEG) & $0-8$ \\
\hline Sowell et al., 2004 & 2004 & $45 / 45$ & $\sim 2$ & WISC (vocab \& block design) & $\begin{array}{l}\text { Cortical thickness, brain } \\
\text { volume }\end{array}$ & $5-11$ \\
\hline Shaw et al., 2006 & 2006 & $307 / 178 / 92$ & $\sim 2$ & WPPSI-III, WISC-III, WAIS-III & Cortical thickness & $4-25$ \\
\hline Brans et al., 2010 & 2010 & $242 / 183$ & 5 & WAIS-III & $\begin{array}{l}\text { Cortical thickness, brain } \\
\text { volume }\end{array}$ & $20-40$ \\
\hline Ramsden et al., 2011 & 2011 & $33 / 33$ & $\sim 3.5$ & WISC-III (wave 1), WAIS-III (wave 2) & Functional \& structural MRI & $12-20$ \\
\hline Tamnes et al., 2013 & 2013 & 79 & & Verbal working memory & Grey matter volume & $8-22$ \\
\hline Burgaleta et al., 2014 & 2014 & $188 / 188$ & $\sim 2$ & WASI & $\begin{array}{l}\text { Cortical thickness, cortical } \\
\text { surface area }\end{array}$ & $6-22$ \\
\hline Evans et al., 2015 & 2015 & $43 / 43 / 12 / 7$ & $\sim 1$ & $\begin{array}{l}\text { WASI, WIAT-II, digit recall, block recall, count } \\
\text { recall, backward digit recall }\end{array}$ & $\begin{array}{l}\text { Brain volume, resting state } \\
\text { connectivity (fMRI) }\end{array}$ & $8-14$ \\
\hline Koenis et al., 2015 & 2015 & $162 / 162$ & $\sim 3$ & WISC-III & $\begin{array}{l}\text { Fractional anisotropy, } \\
\text { streamline count }\end{array}$ & $9-15$ \\
\hline Schnack et al., 2015 & 2015 & $504 / 504$ & $\sim 4$ & WISC-III, short form WAIS \& WAIS-III-NL & $\begin{array}{l}\text { Cortical thickness, cortical } \\
\text { surface area }\end{array}$ & $9-60$ \\
\hline Deoni et al., 2016 & 2016 & $257 / 126 / 39 / 15 / 4$ & $\sim 0.75$ & Mullen Scales of Early Learning & $\begin{array}{l}\text { DWI Myelin water Fraction } \\
\text { (MWF) }\end{array}$ & $0-5$ \\
\hline Wendelken et al., 2017 & 2017 & $523 / 223$ & $\sim 1.5$ & WASI Matrix Reasoning & $\begin{array}{l}\text { Fractional anisotropy, } \\
\text { functional connectivity (fMRI) }\end{array}$ & $6-22$ \\
\hline $\begin{array}{l}\text { Khundrakpam et al., } \\
2017\end{array}$ & 2017 & $306 / ? / ?$ & 2 & WASI & Cortical thickness & $6-18$ \\
\hline Young et al., 2017 & 2017 & $75 / 39 / 18 / 29$ & $\sim 5$ & WPPSI-III & Fractional anisotropy & $0-29$ \\
\hline Román et al., 2018 & 2018 & $132 / 132 / 132$ & $\sim 2$ & WASI & $\begin{array}{l}\text { Cortical thickness, cortical } \\
\text { surface area }\end{array}$ & $6-21$ \\
\hline Tamnes et al., 2018 & 2018 & $237 / 224 / 217$ & $\sim 2$ & WISC \& WASI & Brain volume & $8-29$ \\
\hline Ferrer, 2018 & 2018 & 201/121/71 & $\sim 1.5$ & $\begin{array}{l}\text { WISC-R, Woodcock-Johnson Tests of } \\
\text { Achievement (WJ-R) }\end{array}$ & $\begin{array}{l}\text { Fractional anisotropy, white } \\
\text { matter volume }\end{array}$ & $5-21$ \\
\hline Koenis et al., 2018 & 2018 & $310 / 255 / 130$ & $\sim 3 / \sim 5$ & WISC-III, WAIS-III & Fractional anisotropy & $9-23$ \\
\hline Jaekel et al., 2019 & 2019 & 401 & $\sim 1 / 5$ & K-ABC (ages 6-8) \& WAIS (age 26) & Head circumference & $0-26$ \\
\hline Estrada et al., 2019 & 2019 & $430 / 430 / 430$ & $\sim 2$ & WASI & $\begin{array}{l}\text { Cortical thickness and surface } \\
\text { area }\end{array}$ & $6-22$ \\
\hline Schmitt et al., 2019 & 2019 & $\begin{array}{l}813 / \text { ? (up to } 8 \text { waves, } \\
\text { total } 1748 \text { scans) }\end{array}$ & $\sim 3$ & WPPSI-III, WISC-R, WAIS & Cortical thickness & 3-34 \\
\hline Hahn et al., 2019 & 2019 & $36 / 36$ & 6 & WISC, WAIS & EEG sleep spindles & 8-18 \\
\hline
\end{tabular}

Table 1. An overview of longitudinal studies of brain structure, function and intelligence. For each study we show the abbreviated reference and details about cognitive and imaging measures used. If papers were ambiguous (e.g. only report SD instead of range), numbers reflect an informed estimate. 


\section{Aging}

For a more complete understanding of lifespan trajectories of cognitive abilities and brain structure, we must study, compare and contrast findings from both ends of the lifespan (Tamnes, Walhovd, Dale, et al., 2013). In other words, it is crucial that we investigate not just how brain measures are associated with changes in intelligence in childhood, adolescence and early adulthood, but also the mirroring patterns in later life decline. A recent review (Oschwald et al., 2019) has provided a comprehensive overview of truly longitudinal investigations of age-related decline in cognitive ability and concurrent changes in brain structure. We will here summarize the key findings in the realm of intelligence specifically, but refer the reader to that resource for a more in-depth discussion of the key papers in this field.

Oschwald et al. identified 31 papers that had comprehensive assessments of cognitive performance as well as neural measurements on multiple occasions. The emerging findings across measures of grey matter volume, white matter volume, white matter microstructure (e.g. FA/MD) and more global measures of brain structure (e.g. intra-cranial volume, total brain volume or head size) converged on a series of findings. The most common pattern was that of correlated change, and the overwhelming majority of such findings (exceptions include Bender, Prindle, Brandmaier, \& Raz, 2015) was as expected: more rapid grey matter atrophy, white matter volume decline and/or white matter microstructure loss were associated with more rapid cognitive decline. A second pattern of findings centred on level-change associations between domains. In other words, to what extent does cognitive decline depend on the current state of brain anatomy, and vice versa. Although not all studies used methodology that allowed for such conclusions, many observed a pattern consistent with the hypothesis we term structural scaffolding: The current state of the brain more strongly predicts the rate of cognitive decline than vice versa. One of the earliest papers to observe this was (McArdle et al., 2004), who demonstrated that current ventricle size explained rate of memory decline, above and beyond contemporaneous measures of age and memory score. Both these findings (correlated change and structural scaffolding) are, in turn, in line with the notion of brain maintenance (Nyberg, Lövdén, Riklund, Lindenberger, \& Bäckman, 2012): The main way to maintain current cognitive performance or decelerate cognitive decline is to maintain, to the greatest extent possible, the current state of the brain. Future work should work towards the formalized integration of theories about neurocognitive development throughout the lifespan.

\section{Summary}

In this chapter we provide an overview of studies that investigate co-occurring changes in intelligence, brain structure and brain function from early childhood to early adulthood. From this literature, a few clear conclusions can be drawn. First and foremost, there is a profound sparsity of truly longitudinal work in this field. Regardless of one's precise inclusion criteria, there are currently more studies on fMRI in dogs (Thompkins, Deshpande, Waggoner, \& Katz, 2016 and several studies since) than there are longitudinal investigations of changes in intelligence and brain structure in childhood. This is, perhaps, not entirely surprising: Large, 
longitudinal studies are demanding of time, person power and resources. Combined with several challenges unique to this work, such as updates to MRI scanners, the studies that do exist are all the more impressive. However, there is a recent and rapid increase in large longitudinal studies - As Table 1 shows, almost half of all studies discussed date from just the last three years, suggesting many more studies will emerge in the near future. Taken together, although not plentiful in number, the studies so far allow several conceptual and substantive conclusions.

\section{Timing matters}

First and foremost, timing matters. All metrics used in the studies above, from grey matter volume and thickness to structural and functional connectivity change rapidly, non-linearly and in complex manners. One key consequence of these changes is that the cross-sectional associations between brain measures and intelligence will be heavily dependent on the age and age distribution of the population being studied. As multiple studies above show, measures such as cortical thickness can show positive, no or negative correlations with intelligence during development, even in the same cohort, in children of different ages. This alone should send a clear message to developmental cognitive neuroscientists: Age, as much as gender, country of origin and/or SES affects the nature of the associations we might observe.

In studies that incorporate quantitative parameters of change between brain and behaviour, two findings emerge across multiple studies. First, correlated change between brain structure and intelligence. Given the same interval, individuals who demonstrate greater gains in intelligence often show more rapid changes in structural development as well. Multiple, complementary explanations for such observations can exist. One is that changes in both domains are governed by some third variable, such as the manifestation of a complex pattern of gene expression at a particular maturational period. A methodological explanation of the same statistical effect is that the temporal resolution of a given study is not suitable to tease apart lead-lag distinctions that may in fact exist - Although in reality cognitive ability may precede brain change or vice versa, the actual intervals used in studies (often multiple years) will obscure the fine-grained temporal unfolding. This second pattern observed across multiple cohorts is what we referred to above as 'structural scaffolding'. This is the finding that current brain states (as indexed by measures of brain structure) tend to govern (statistically) the rate of change in cognitive performance. This pattern is observed in multiple studies (e.g. Ferrer, 2018; Wendelken et al., 2017), and is consistently in the same direction: 'Better' brain structure is generally associated with greater gains (in children) or shallower declines (in older individuals) than individuals with lower scores on brain structural metrics. This pattern is highly intriguing and worth further study, as it may have profound implications for how we think about the relationship between the brain and intelligence. However, this parameter can only be observed by studies that implement methodology (such as the latent change score model) which allows for the quantification of this pattern. This illustrates the importance of suitable quantitative methodology. 


\section{Methods matter}

It is impossible to provide a precise quantitative meta-analysis of the findings in this field, as the analytical choices govern which parameters of interest are estimated and report.

Different models may provide somewhat, or even very, different conclusions when applied to different, or even the same, datasets (Oschwald et al., 2019). There is no single solution to this challenge: Particular methods have strengths and weaknesses, making them more or less suitable for the particular topic being studied. Simultaneously, it seems clear that often (but not always), the choice of methodology is governed as much by conventions of the (sub)field and software limitations as by considered analytical choices. A broader awareness of the range of suitable methods out there would likely improve this state of affairs. A recent special issue (Pfeifer, Allen, Byrne, \& Mills, 2018) brings together a wide range of innovations, perspectives and methodological challenges, providing an excellent starting point for researchers looking to expand their horizons. Another line of improvement would be the strengthening of the explanatory theoretical frameworks used to conceptualize the development of intelligence. Studies are now of sufficient complexity and richness that we can, and should, move beyond the reporting of simple bivariate associations, and develop testable theoretical frameworks that bring together the disparate yet fascinating findings from cognitive development, genetics, grey and white matter and brain function. Most crucially, such theories should be guided by the promise of translation into tractable quantitative models, such that others may build, refine or replace models.

The longitudinal neuroscience of intelligence in childhood and adolescence is only in its infancy, yet many exciting findings have already been made. Many discoveries await for a field willing and able to collaboratively work towards a cumulative developmental cognitive neuroscience of intelligence across the entire lifespan. 


\section{References}

Barbey, A. K., Colom, R., Paul, E. J., \& Grafman, J. (2014). Architecture of fluid intelligence and working memory revealed by lesion mapping. Brain Structure \& Function, 219(2), 485-494. https://doi.org/10.1007/s00429-013-0512-z

Basten, U., Hilger, K., \& Fiebach, C. J. (2015). Where smart brains are different: A quantitative meta-analysis of functional and structural brain imaging studies on intelligence. Intelligence, 51, 10-27. https://doi.org/10.1016/j.intell.2015.04.009

Beckwith, L., \& Parmelee, A. H. (1986). EEG Patterns of Preterm Infants, Home Environment, and Later IQ. Child Development, 57(3), 777-789. https://doi.org/10.2307/1130354

Bender, A. R., Prindle, J. J., Brandmaier, A. M., \& Raz, N. (2015). White matter and memory in healthy adults: Coupled changes over two years. Neurolmage. https://doi.org/10.1016/j.neuroimage.2015.10.085

Bengtsson, S. L., Nagy, Z., Skare, S., Forsman, L., Forssberg, H., \& Ullén, F. (2005). Extensive piano practicing has regionally specific effects on white matter development. Nature Neuroscience, 8(9), 1148-1150. https://doi.org/10.1038/nn1516

Borchers, L. R., Bruckert, L., Dodson, C. K., Travis, K. E., Marchman, V. A., Ben-Shachar, M., \& Feldman, H. M. (2019). Microstructural properties of white matter pathways in relation to subsequent reading abilities in children: a longitudinal analysis. Brain Structure and Function, 224(2), 891-905.

Brans, R. G. H., Kahn, R. S., Schnack, H. G., Baal, G. C. M. van, Posthuma, D., Haren, N. E. M. van, ... Pol, H. E. H. (2010). Brain Plasticity and Intellectual Ability Are Influenced by Shared Genes. Journal of Neuroscience, 30(16), 5519-5524. https://doi.org/10.1523/JNEUROSCI.5841-09.2010

Burgaleta, M., Johnson, W., Waber, D. P., Colom, R., \& Karama, S. (2014). Cognitive ability changes and dynamics of cortical thickness development in healthy children and adolescents. Neurolmage, 84, 810-819.

https://doi.org/10.1016/j.neuroimage.2013.09.038

Deary, I. J., Penke, L., \& Johnson, W. (2010). The neuroscience of human intelligence differences. Nature Reviews Neuroscience, 11(3), 201-211. https://doi.org/10.1038/nrn2793

Deoni, S. C. L., O'Muircheartaigh, J., Elison, J. T., Walker, L., Doernberg, E., Waskiewicz, N., ... Jumbe, N. L. (2016). White matter maturation profiles through early childhood predict general cognitive ability. Brain Structure \& Function, 221, 1189-1203. https://doi.org/10.1007/s00429-014-0947-x

Dickens, W. T., \& Flynn, J. R. (2001). Heritability Estimates Versus Large Environmental Effects: The IQ Paradox Resolved. Psychological Review, 108(2), 346-369. https://doi.org/10.1037//0033-295X

Estrada, E., Ferrer, E., Román, F. J., Karama, S., \& Colom, R. (2019). Time-lagged associations between cognitive and cortical development from childhood to early adulthood. Developmental Psychology, 55(6), 1338-1352. https://doi.org/10.1037/dev0000716

Evans, A. C., \& Brain Development Cooperative Group. (2006). The NIH MRI study of normal brain development. Neuroimage, 30(1), 184-202. 
Evans, T. M., Kochalka, J., Ngoon, T. J., Wu, S. S., Qin, S., Battista, C., \& Menon, V. (2015). Brain Structural Integrity and Intrinsic Functional Connectivity Forecast 6 Year Longitudinal Growth in Children's Numerical Abilities. Journal of Neuroscience, 35(33), 11743-11750. https://doi.org/10.1523/JNEUROSCI.0216-15.2015

Ferrer, E. (2018). Discrete- and semi-continuous time latent change score models of fluid reasoning development from childhood to adolescence. In S. M. Boker, K. J. Grimm, \& E. Ferrer (Eds.), Longitudinal Multivariate Psychology. Routledge.

Ferrer, E., \& McArdle, J. J. (2004). An Experimental Analysis of Dynamic Hypotheses About Cognitive Abilities and Achievement From Childhood to Early Adulthood. Developmental Psychology, 40(6), 935-952.

Ferrer, E., Shaywitz, B. A., Holahan, J. M., Marchione, K., \& Shaywitz, S. E. (2010). Uncoupling of reading and IQ over time: Empirical evidence for a definition of dyslexia. Psychological Science, 21(1), 93-101. https://doi.org/10.1177/0956797609354084

Ferrer, E., Whitaker, K. J., Steele, J. S., Green, C. T., Wendelken, C., \& Bunge, S. A. (2013). White matter maturation supports the development of reasoning ability through its influence on processing speed. Developmental Science, 16(6), 941-951. https://doi.org/10.1111/desc.12088

Grimm, K. J., An, Y., McArdle, J. J., Zonderman, A. B., \& Resnick, S. M. (2012). Recent changes leading to subsequent changes: Extensions of multivariate latent difference score models. Structural Equation Modeling: A Multidisciplinary Journal, 19(March 2015), 268-292. https://doi.org/10.1080/10705511.2012.659627

Gross, C. (1995). Aristotle on the Brain. The Neuroscientist. https://doi.org/10.1177/107385849500100408

Hahn, M., Joechner, A., Roell, J., Schabus, M., Heib, D. P., Gruber, G., ... Hoedlmoser, K. (2019). Developmental changes of sleep spindles and their impact on sleepdependent memory consolidation and general cognitive abilities: A longitudinal approach. Developmental Science, 22(1). https://doi.org/10.1111/desc.12706

Huarte, J. Examen de ingenios (1594). The examination of mens wits. By John Huarte. Translated out of the Spanish tongue by M. Camillo Camilli. Englished out of his Italian, by R.C. Esquire London: Printed by Adam Islip, for C. Hunt of Excester, 1594

Jaekel, J., Sorg, C., Baeuml, J., Bartmann, P., \& Wolke, D. (2019). Head Growth and Intelligence from Birth to Adulthood in Very Preterm and Term Born Individuals. Journal of the International Neuropsychological Society, 25(1), 48-56. https://doi.org/10.1017/S135561771800084X

Jensen, A. R. (1998). The $g$ Factor: The Science of Mental Ability. Praeger.

Jones, D. K., Knösche, T. R., \& Turner, R. (2013). White matter integrity, fiber count, and other fallacies: The do's and don'ts of diffusion MRI. Neurolmage, 73, 239-254. https://doi.org/10.1016/j.neuroimage.2012.06.081

Judd, N., Sauce, B., Wiedenhoeft, J., Tromp, J., Chaarani, B., Schliep, A., ... \& Becker, A. (2020). Cognitive and brain development is independently influenced by socioeconomic status and polygenic scores for educational attainment. bioRxiv, 866624. 
Jung, R. E., \& Haier, R. J. (2007). The Parieto-Frontal Integration Theory (P-FIT) of intelligence: Converging neuroimaging evidence. Behavioral and Brain Sciences, 30(02), 135. https://doi.org/10.1017/S0140525X07001185

Kail, R. V. (1998). Speed of information processing in patients with multiple sclerosis. Journal of Clinical and Experimental Neuropsychology, 20(1), 98-106. https://doi.org/10.1076/jcen.20.1.98.1483

Khundrakpam, B. S., Lewis, J. D., Reid, A., Karama, S., Zhao, L., Chouinard-Decorte, F., \& Evans, A. C. (2017). Imaging structural covariance in the development of intelligence. Neurolmage, 144, 227-240. https://doi.org/10.1016/j.neuroimage.2016.08.041

Kievit, R. A., Hofman, A. D., \& Nation, K. (2019). Mutualistic Coupling Between Vocabulary and Reasoning in Young Children: A Replication and Extension of the Study by Kievit et al. (2017). Psychological Science, 0956797619841265. https://doi.org/10.1177/0956797619841265

Kievit, R. A., Lindenberger, U., Goodyer, I. M., Jones, P. B., Fonagy, P., Bullmore, E. T., ... Dolan, R. J. (2017). Mutualistic coupling between vocabulary and reasoning supports cognitive development during late adolescence and early adulthood. Psychological Science.

Kievit, R. A., Brandmaier, A. M., Ziegler, G., Van Harmelen, A. L., de Mooij, S. M., Moutoussis, M., ... \& Lindenberger, U. (2018). Developmental cognitive neuroscience using latent change score models: A tutorial and applications. Developmental cognitive neuroscience, 33, 99-117.

Koenis, M. M. G., Brouwer, R. M., Heuvel, M. P. van den, Mandl, R. C. W., Soelen, I. L. C. van, Kahn, R. S., ... Pol, H. E. H. (2015). Development of the brain's structural network efficiency in early adolescence: A longitudinal DTI twin study. Human Brain Mapping, 36(12), 4938-4953. https://doi.org/10.1002/hbm.22988

Koenis, M. M. G., Brouwer, R. M., Swagerman, S. C., Soelen, I. L. C. van, Boomsma, D. I., \& Pol, H. E. H. (2018). Association between structural brain network efficiency and intelligence increases during adolescence. Human Brain Mapping, 39(2), 822-836. https://doi.org/10.1002/hbm.23885

McArdle, J. J., Hamgami, F., Jones, K., Jolesz, F., Kikinis, R., Spiro, A., \& Albert, M. S. (2004). Structural modeling of dynamic changes in memory and brain structure using longitudinal data from the normative aging study. The Journals of Gerontology. Series B, Psychological Sciences and Social Sciences, 59(6), P294-304. https://doi.org/10.1093/GERONB/59.6.P294

Neubauer, A. C., \& Fink, A. (2009). Intelligence and neural efficiency: Measures of brain activation versus measures of functional connectivity in the brain. Intelligence, 37(2), 223-229. https://doi.org/10.1016/j.intell.2008.10.008

Nyberg, L., Lövdén, M., Riklund, K., Lindenberger, U., \& Bäckman, L. (2012). Memory aging and brain maintenance. Trends in Cognitive Sciences, 16(5), 292-305. https://doi.org/10.1016/j.tics.2012.04.005

Oschwald, J., Guye, S., Liem, F., Rast, P., Willis, S., Röcke, C., ... Mérillat, S. (2019). Brain structure and cognitive ability in healthy aging: A review on longitudinal correlated 
change. Reviews in the Neurosciences, O(0). https://doi.org/10.1515/revneuro-20180096

Peng, P., \& Kievit, R. (2019). The Development of Academic Achievement and Cognitive Abilities: A Mutualistic Perspective [Preprint]. https://doi.org/10.31219/osf.io/9u86q

Peng, P., Wang, T., Wang, C., \& Lin, X. (2019). A Meta-Analysis on the Relation Between Fluid Intelligence and Reading/Mathematics: Effects of Tasks, Age, and Social Economics Status. Psychological Bulletin, 145(2), 189-236. https://doi.org/10.1037/bul0000182

Pfeifer, J. H., Allen, N. B., Byrne, M. L., \& Mills, K. L. (2018). Modeling Developmental Change: Contemporary Approaches to Key Methodological Challenges in Developmental Neuroimaging. Developmental Cognitive Neuroscience, 33, 1-4. https://doi.org/10.1016/j.dcn.2018.10.001

Pietschnig, J., Penke, L., Wicherts, J. M., Zeiler, M., \& Voracek, M. (2015). Meta-analysis of associations between human brain volume and intelligence differences: How strong are they and what do they mean? Neuroscience \& Biobehavioral Reviews, 57, 411432. https://doi.org/10.1016/j.neubiorev.2015.09.017

Ramsden, S., Richardson, F. M., Josse, G., Thomas, M. S. C., Ellis, C., Shakeshaft, C., ... Price, C. J. (2011). Verbal and non-verbal intelligence changes in the teenage brain. Nature, 479(7371), 113-116. https://doi.org/10.1038/nature10514

Raz, N., \& Lindenberger, U. (2011). Only Time Will Tell: Cross-Sectional Studies Offer No Solution to the Age - Brain - Cognition Triangle: Comment on Salthouse ( 2011 ). 137(5), 790-795. https://doi.org/10.1037/a0024503

Ritchie, S. J., Quinlan, E. B., Banaschewski, T., Bokde, A. L., Desrivieres, S., Flor, H., ... \& Ittermann, B. (2019). Neuroimaging and genetic correlates of cognitive ability and cognitive development in adolescence. Psyarxiv, https://psyarxiv.com/8pwd6/

Rocca, J. (2009). Galen and the ventricular system. Journal of the History of the Neurosciences, 6(3). Retrieved from https://www.tandfonline.com/doi/abs/10.1080/09647049709525710?casa_token=u aaDpevYWpgAAAAA:YgJ2sfv80R1vUd6M0VIqfxFd6hkCxAsKhim1_BtZuwPHteZ4Wmwah5FWBCINOkHCi3L97VL1zuDiqo

Román, F. J., Morillo, D., Estrada, E., Escorial, S., Karama, S., \& Colom, R. (2018). Brainintelligence relationships across childhood and adolescence: A latent-variable approach. Intelligence, 68, 21-29. https://doi.org/10.1016/j.intell.2018.02.006

Schmitt, J. E., Raznahan, A., Clasen, L. S., Wallace, G. L., Pritikin, J. N., Lee, N. R., ... Neale, M. C. (2019). The Dynamic Associations Between Cortical Thickness and General Intelligence are Genetically Mediated. Cerebral Cortex. https://doi.org/10.1093/cercor/bhz007

Schnack, H. G., van Haren, N. E. M., Brouwer, R. M., Evans, A., Durston, S., Boomsma, D. I., ... Hulshoff Pol, H. E. (2015). Changes in Thickness and Surface Area of the Human Cortex and Their Relationship with Intelligence. Cerebral Cortex, 25(6), 1608-1617. https://doi.org/10.1093/cercor/bht357 
Shaw, P., Greenstein, D., Lerch, J., Clasen, L., Lenroot, R., Gogtay, N., ... Giedd, J. (2006). Intellectual ability and cortical development in children and adolescents. Nature, 440(7084), 676-679. https://doi.org/10.1038/nature04513

Sowell, E. R., Thompson, P. M., Leonard, C. M., Welcome, S. E., Kan, E., \& Toga, A. W. (2004). Longitudinal Mapping of Cortical Thickness and Brain Growth in Normal Children. Journal of Neuroscience, 24(38), 8223-8231. https://doi.org/10.1523/JNEUROSCI.1798-04.2004

Spearman, C. (1904). "General Intelligence," Objectively Determined and Measured. The American Journal of Psychology, 15(2), 201-292. https://doi.org/10.2307/1412107

Tamnes, C. K., Bos, M. G. N., van de Kamp, F. C., Peters, S., \& Crone, E. A. (2018). Longitudinal development of hippocampal subregions from childhood to adulthood. Developmental Cognitive Neuroscience, 30, 212-222. https://doi.org/10.1016/j.dcn.2018.03.009

Tamnes, C. K., Walhovd, K. B., Dale, A. M., Østby, Y., Grydeland, H., Richardson, G., ... Fjell, A. M. (2013). Brain development and aging: Overlapping and unique patterns of change. Neurolmage, 68, 63-74. https://doi.org/10.1016/j.neuroimage.2012.11.039

Tamnes, C. K., Walhovd, K. B., Grydeland, H., Holland, D., Østby, Y., Dale, A. M., \& Fjell, A. M. (2013). Longitudinal working memory development is related to structural maturation of frontal and parietal cortices. Journal of Cognitive Neuroscience, 25(10), 1611-1623. https://doi.org/10.1162/jocn_a_00434

Thompkins, A. M., Deshpande, G., Waggoner, P., \& Katz, J. S. (2016). Functional Magnetic Resonance Imaging of the Domestic Dog: Research, Methodology, and Conceptual Issues. Comparative Cognition \& Behavior Reviews, 11, 63-82. https://doi.org/10.3819/ccbr.2016.110004

Volkow, N. D., Koob, G. F., Croyle, R. T., Bianchi, D. W., Gordon, J. A., Koroshetz, W. J., ... Weiss, S. R. B. (2018). The conception of the ABCD study: From substance use to a broad NIH collaboration. Developmental Cognitive Neuroscience, 32, 4-7. https://doi.org/10.1016/j.dcn.2017.10.002

Wandell, B. A. (2016). Clarifying Human White Matter. Annual Review of Neuroscience, aop.

Wendelken, C., Ferrer, E., Ghetti, S., Bailey, S. K., Cutting, L., \& Bunge, S. A. (2017). Frontoparietal Structural Connectivity in Childhood Predicts Development of Functional Connectivity and Reasoning Ability: A Large-Scale Longitudinal Investigation. The Journal of Neuroscience: The Official Journal of the Society for Neuroscience, 37(35), 8549-8558. https://doi.org/10.1523/JNEUROSCI.3726-16.2017

Wenger, E., Brozzoli, C., Lindenberger, U., \& Lövdén, M. (2017). Expansion and Renormalization of Human Brain Structure During Skill Acquisition. Trends in Cognitive Sciences, 21(12), 930-939. https://doi.org/10.1016/j.tics.2017.09.008

Widaman, K. F., Ferrer, E., \& Conger, R. D. (2010). Factorial Invariance within Longitudinal Structural Equation Models: Measuring the Same Construct across Time. Child Development Perspectives, 4(1), 10-18. https://doi.org/10.1111/j.17508606.2009.00110.x 
Young, J. M., Morgan, B. R., Whyte, H. E. A., Lee, W., Smith, M. L., Raybaud, C., ... Taylor, M.

J. (2017). Longitudinal Study of White Matter Development and Outcomes in Children Born Very Preterm. Cerebral Cortex, 27(8), 4094-4105.

https://doi.org/10.1093/cercor/bhw221 